\title{
Silver Nanocubes Coated in Ceria: Core/Shell Size Effects on Light- Induced Charge Transfer
}

\author{
Devin B. O’Neill, ${ }^{\dagger} \odot$ Daniel Prezgot, ${ }^{\S}$ Anatoli Ianoul, ${ }^{\S \odot ~ C e e s ~ O t t o, ~}{ }^{\ddagger}$ Guido Mul, ${ }^{\dagger \odot}$ \\ and Annemarie Huijser* ${ }^{*} \odot \odot$
}

${ }^{\dagger}$ PhotoCatalytic Synthesis Group, MESA+ Institute for Nanotechnology, and ${ }^{\ddagger}$ Medical Cell BioPhysics Group, MESA+ Institute for Nanotechnology, University of Twente, P.O. Box 217, 7500 AE Enschede, The Netherlands

${ }^{\S}$ Department of Chemistry, Carleton University, Ottawa, ON K1S 5B6, Canada

\section{Supporting Information}

ABSTRACT: Plasmonic sensitization of semiconductors is an attractive approach to increase light-induced photocatalytic performance; one method is to use plasmonic nanostructures in core@shell geometry. The occurrence and mechanism of synergetic effects in photocatalysis of such geometries are under intense debate and proposed to occur either through light-induced charge transfer (CT) or through thermal effects. This study focuses on the relation between the dimensions of $\mathrm{Ag} @ \mathrm{CeO}_{2}$ nanocubes, the wavelength-dependent efficiency, and the mechanism of light-induced direct CT. A 4mercaptobenzoic acid (4-MBA) linker between core and shell acts as a Raman probe for CT. For all $\mathrm{Ag} @ \mathrm{CeO}_{2}$ nanocubes, CT increases with

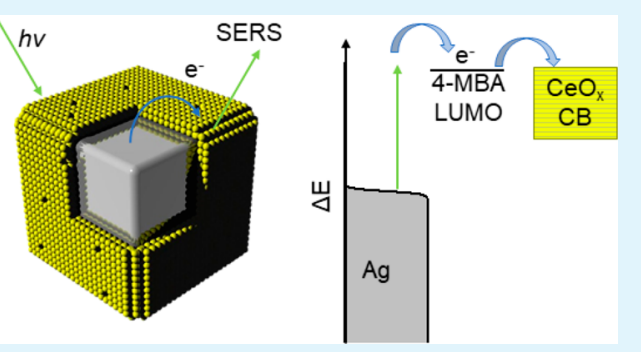
decreasing excitation wavelength, with notable increase at and below $514 \mathrm{~nm}$. This is fully explainable by CT from silver to the 4-MBA LUMO, with the increase for excitation wavelengths that exceed the Ag/4-MBA LUMO gap of $2.28 \mathrm{eV}$ (543 nm). A second general trend observed is an increase in CT yield with ceria shell thickness, which is assigned to relaxation of the excited electron further into the ceria conduction band, potentially producing defects.

KEYWORDS: plasmonic, sensitized photocatalyst, ceria, silver nanocube, Raman spectroscopy, FDTD, light-induced charge transfer

\section{INTRODUCTION}

Sensitized photocatalysts are gaining increasing attention with the growing concerns about the state of the environment and, with it, the desire to use more sustainable energy sources. Nanostructures that support local surface plasmon resonances (LSPR), which is the photoinduced collective oscillation of electrons, can be used to sensitize semiconductors through decoration, incorporation, or hierarchical construction such as core@shell particles. ${ }^{1-3}$ Core@shell nanostructures are attractive because they offer controllable overlap of the enhanced electric field with the semiconductor and even the boundary of the semiconductor with the external media by controlling the thickness of the shell.

Plasmonic enhancement of photocatalysts can be divided into four mechanisms: plasmon-induced resonance energy transfer (PIRET), ${ }^{4-6}$ direct charge transfer (CT) during photoexcitation or before plasmon dephasing, indirect CT after plasmon dephasing, ${ }^{4,7-9}$ and as a local heat source. ${ }^{10}$ PIRET requires coupling of transition dipoles and is strongly dependent on the distance and spectral overlap of the plasmonic donor and the energy acceptor but can occur through space. ${ }^{4,5}$ Unlike PIRET, both CT mechanisms need electronic coupling. Direct CT can be divided into three categories based on wavelength dependence and association with plasmonic modes. (1) Plasmon-induced interfacial CT transition (PICTT): a new electronic transition that requires strong coupling between the donor and acceptor across the interface of the plasmonic particle and semiconductor. (2) Direct interfacial CT transition (DICTT): a CT transition not associated with a plasmonic mode, but instead with a weaker metal-adsorbate transition possible above a threshold photon energy. ${ }^{8}$ (3) Chemical interface dampening (CID): a new adsorbate-induced plasmon dephasing pathway, with a quantum yield in agreement with the LSPR absorption. ${ }^{8,11}$ For indirect CT there is a sensitivity to the photon energy as excited electrons must overcome the Schottky barrier prior to relaxation through heat generation in the lattice. ${ }^{4,5,7-10}$

As well as acting as a sensitizer, ${ }^{2,3}$ the LSPR is also a sensor for the local environment: changes to the local refractive index $(n)$ cause spectral shifts ${ }^{12}$ and enhance Raman intensities. ${ }^{13,14}$ The LSPR spectral properties depend on the particle material, shape, size, and local environment. ${ }^{15,16}$ Silver nanocubes support several LSPR modes; the most relevant to this study is the dominant corner mode (red-most mode for an isolated cube). This mode projects the furthest from the surface and is most sensitive to the local refractive index for supported cubes. ${ }^{12,15-17}$ The distance-dependent refractive index sensitivity (RIS $(t)$, with $t$ being the distance), determined from finite-domain time-

Received: October 10, 2019

Accepted: December 9, 2019

Published: December 9, 2019 

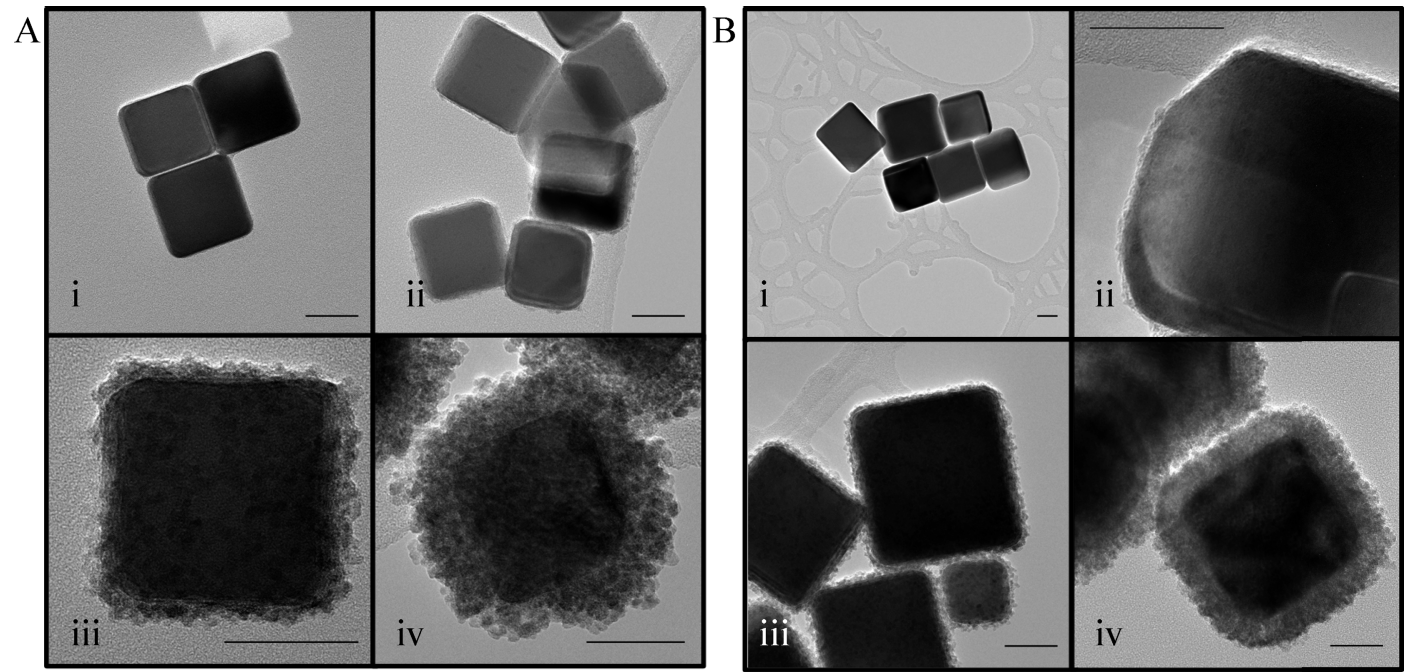

Figure 1. TEM of (A) $47 \pm 7 \mathrm{~nm}$ silver cubes with (i) no shell, (ii) incomplete coverage with $2.6 \pm 0.3 \mathrm{~nm} \mathrm{CeO}{ }_{2}$ crystallites, (iii) a $4.6 \pm 1.3 \mathrm{~nm}$ shell, and (iv) $14.1 \pm 3.9 \mathrm{~nm}$ shell and (B) $71 \pm 9 \mathrm{~nm}$ cubes with (i) no shell, (ii) incomplete coverage with $2.3 \pm 0.2 \mathrm{~nm}$ crystallites, (iii) a $3.7 \pm 1.1 \mathrm{~nm}$ ceria shell, and (iv) a $17.6 \pm 3.5 \mathrm{~nm}$ conformal shell. The scale bars in all micrographs are $30 \mathrm{~nm}$. Core and shell dimensions have all been determined from no fewer than 20 measurements. Additional micrographs are presented in Figure S3.

difference (FDTD) simulations, allows for determination of the shell coverage.

$\mathrm{CeO}_{2}$ is a well-known catalyst with demonstrable potential as a photocatalyst, ${ }^{18-22}$ which supports $\mathrm{Ce}$ (IV) and Ce(III) states forming reactive oxygen vacancies ${ }^{23,24}$ and catalyzes reactions such as reducing $\mathrm{CO}_{2}$ into $\mathrm{CO} .{ }^{25} \mathrm{CeO}_{2}$ has a bulk direct band gap of $3.6 \mathrm{eV}$ and an indirect band gap of $\sim 2.4 \mathrm{eV} ;{ }^{26}$ in addition, trap states may induce light absorption throughout the visible regime. Silver nanoparticles are an attractive choice to sensitize ceria as they have high quality plasmonic modes, ${ }^{27}$ a high refractive index sensitivity, ${ }^{28}$ and, through geometric control, light absorption throughout the visible and near-infrared regime. ${ }^{29}$ Additionally, use of a core@shell geometry is of interest, as the metal core can be isolated-either to prevent reactions, such as silver dissolving, or to promote selectivity by only having the semiconductor as an available active site. There have been studies on $\mathrm{Ag} / \mathrm{CeO}_{2}$ (nano) composites, ${ }^{30-34}$ but only few studies report on core@shell geometries, ${ }^{35-39}$ despite their promise as photocatalysts. Silver nanocubes are attractive because of the spatial localization of modes ${ }^{17}$ and high $|E|^{2}$ enhancement, ${ }^{40}$ while preserving a high degree of symmetry. Generalizable fundamental understanding of structural effects on the photophysics is important, specifically on the occurrence, mechanism, and wavelength dependence of photoinduced CT.

The aim of this work is to investigate photoinduced CT in $\mathrm{Ag} @ \mathrm{CeO}_{2}$ core@shell nanocubes through surface-enhanced Raman scattering (SERS) spectroscopy by using the method developed by Lombardi and Birke. ${ }^{41}$ Comparing the intensities of totally and non-totally symmetric Raman modes allows for a quantification of the relative enhancement from the LSPR and from $\mathrm{CT} .{ }^{41}$ We have investigated the role of core and shell dimensions on CT by using a 4-mercaptobenzoic acid (4-MBA) tag between the silver and ceria as the Raman probe. Here, the wavelength-dependent effects of core and shell dimensions on CT have been examined. FDTD simulations have been performed to model the shell coverage of the $\mathrm{Ag} @ \mathrm{CeO}_{2}$ core@shell nanocubes and determine the distance dependences of the refractive index sensitivity. These give insights into the relationship between $\mathrm{Ag} @ \mathrm{CeO}_{2}$ core@shell nanostructure design and light-induced $\mathrm{CT}$ processes.

\section{MATERIALS AND METHODS}

Synthesis Ag Nanocubes. Ag nanocubes of varied sizes were synthesized by using a method adapted from the polyol synthesis; ${ }^{42,43}$ the solvent for all solutions used in the synthesis was ethylene glycol. A round-bottom flask, containing $40 \mathrm{~mL}$ of ethylene glycol (Fluka, $>99.9 \%$ ) and $0.4 \mathrm{~g}$ of polyvinylpyrrolidone (PVP, Sigma-Aldrich), was heated at $150{ }^{\circ} \mathrm{C}$ for $2 \mathrm{~h}$. $0.4 \mathrm{~mL}$ of $3 \mathrm{mM} \mathrm{Na} 2 \mathrm{~S}$ was then added, followed by the dropwise addition of $2.5 \mathrm{~mL}$ of $280 \mathrm{mM} \mathrm{AgNO}{ }_{3}(\mathrm{Alfa}$ Aesar, 99+\%). The reaction was followed with a spectrometer (Avantis Starline AvaSpec-2048) and quenched by cooling when the desired spectrum was observed. After quenching, the product was cleaned through centrifugation $(9000 \mathrm{~g})$ and redispersion into ethanol through sonication five times. The final suspensions were $10 \mathrm{~mL}$ and had an attenuance of $4 \times 10^{2}$ at the spectral maximum.

Synthesis Ag@CeO 2 Core-Shell Nanocubes. Silver nanocubes with edge lengths of $47 \pm 7$ and $71 \pm 9 \mathrm{~nm}$ were coated with conformal ceria shells of different thicknesses by varying the cerium nitrate and sodium hydroxide concentrations. A mild synthesis method to deposit the ceria shell on the plasmonic cube was chosen, as high temperatures or caustic reagents can easily damage or destroy the particles. ${ }^{44}$

Ceria was deposited on silver in a method adapted from bulk precipitation methods. ${ }^{45} \mathrm{Ce}\left(\mathrm{NO}_{3}\right)_{3} \cdot 6 \mathrm{H}_{2} \mathrm{O}_{(\mathrm{aq})}$ (Sigma-Aldrich, 99\% trace metal) was exposed to a molar equivalent of $\mathrm{NaOH}_{(\mathrm{aq})}$ (SigmaAldrich, $>98 \%$ ) in the presence of 4-MBA-modified Ag nanocubes. Specifically, $1000 \mu \mathrm{L}$ of the OD $\sim 400$ particle suspension was added to

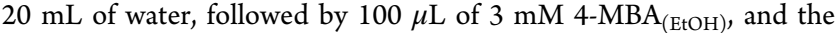
suspension was then incubated for $10 \mathrm{~min}$. The amount of ceria precursor and base to particles was varied to achieve different ceria shell thicknesses, where 10,100 , or $5000 \mu \mathrm{mol}$ of both $\mathrm{Ce}\left(\mathrm{NO}_{3}\right)_{3(\mathrm{aq})}$ and $\mathrm{NaOH}_{(\mathrm{aq})}$ was used. Samples were cleaned through centrifugation $(4000 \mathrm{~g})$ and dispersion into Uvasol ethanol (VWR) three times.

Initial studies did not include 4-MBA, and ceria grew off the PVP on the Ag nanocubes; however, to observe CT the 4-MBA was included. In mildly acidic conditions 4-MBA attaches to silver by the thiol group; ${ }^{46}$ precipitating ceria then binds to the free carboxylic group (similarly, the ceria can grow off the oxygen in $\mathrm{PVP}^{37}$ ) and grows off the $\mathrm{Ag}$ nanocubes. This allows for the 4-MBA to act as a linker and be in electronic contact with both the silver and ceria.

FDTD Simulations. The program Lumerical FDTD Solutions was used for simulations for theoretical support of the experimental spectral changes caused by the shell deposition. Ag nanocubes were modeled with rounded edges and corners $(r=2.5 \mathrm{~nm})$; ceria shells were modeled with the same structure. As thickness was increased, the radii of edges and corners were increased, such that the particle was spherical when 

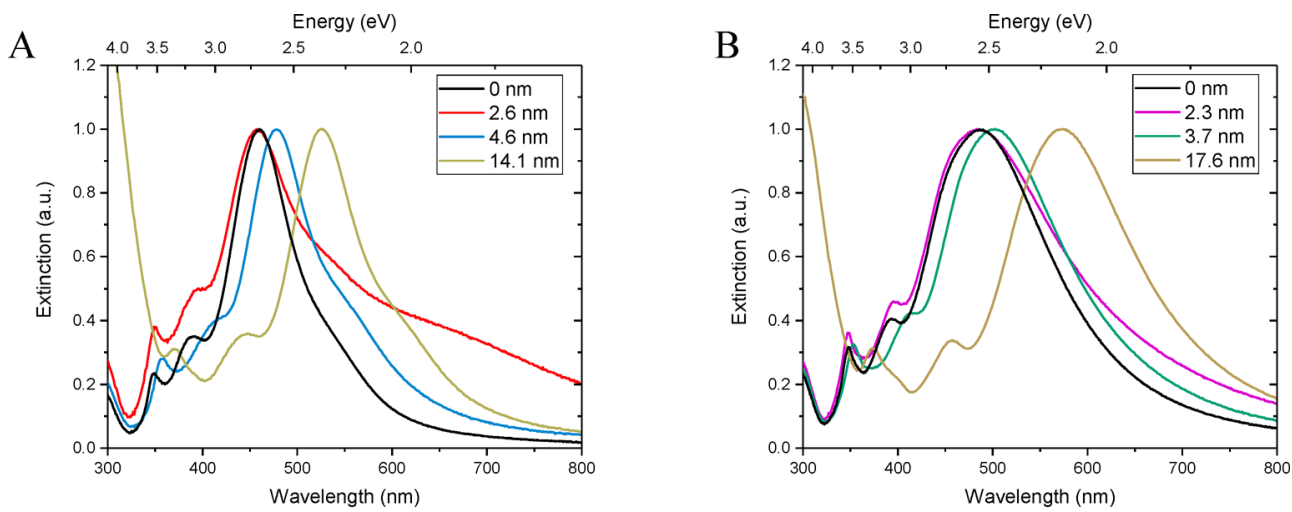

Figure 2. UV-vis spectra of (A) $47 \mathrm{~nm}$ and (B) $71 \mathrm{~nm}$ silver nanocubes, PVP capped ( $0 \mathrm{~nm}$ ), and 4-MBA treated and coated with ceria (shell thickness given in legend), suspended in ethanol. Red-shifting of modes occurs due to the increased local index of refraction caused by the presence of $\mathrm{CeO}_{2}$.

the thickness is equal to the core size. Scattering and absorption were both considered to produce a calculated extinction relatable to experimental results. While the Johnson and Christy ${ }^{47}$ model for silver was used, noncomplex indices of refraction were used to model the shell and surrounding media. The refractive index value $n$ for the ceria shell was set at 2.2, and a value of 1.337 for the solvent (water) was used. Using a fixed value for the shell facilitates generalization of the results for use with the Lorentz-Lorenz equation.

Nanostructure Characterization. The UV-vis spectra of Ag@ $\mathrm{CeO}_{2}$ core-shell nanostructures in suspension were collected by using a ThermoSci EVO600 spectrometer. For refractive index sensitivity measurements, the solvents used were deionized water, ethanol, and ethylene glycol. Characterization of the nanoparticles used a Philips CM300ST-FEG transmission electron microscope (TEM) with acceleration voltage of $300 \mathrm{kV}$ and energy dispersive $\mathrm{X}$-ray analysis (EDX). The dimensions of uncoated Ag nanocubes and $\mathrm{Ag} @ \mathrm{CeO}_{2}$ core-shell nanocubes were determined from 20 measurements of shells/crystallites on the particles. Scanning electron micrographs (SEM) were recorded by using a JSM-6010LA SEM. X-ray diffraction (XRD) analysis was performed on a Bruker D2 $(\mathrm{Cu} \mathrm{K} \alpha$ source $)$ diffractometer, which revealed increased shielding of the silver core with increased shell thickness; the diffractogram is shown in Figure S1 of the Supporting Information. X-ray photoelectron spectroscopy (XPS) was performed with a Quantera SXM, with an Al K $\alpha$ source $(1486.6 \mathrm{eV})$.

Raman Measurements. Raman measurements were performed on a diluted suspension of $\mathrm{Ag} @ \mathrm{CeO}_{2}$ core-shell nanocubes in Milli- $\mathrm{Q}$ water. Raman spectra at $785 \mathrm{~nm}$ excitation were collected with an Avantes AvaRaman probe system, containing 1\% ethanol. For Raman measurements with 458, 488, 514, and $568 \mathrm{~nm}$ excitations, the excitation source was an $\mathrm{Ar} / \mathrm{Kr}$ ion laser (Coherent). A single grating monochromator (Jobin Yvon, focal length $640 \mathrm{~mm}$ ) with a liquid $\mathrm{N}_{2}$ cooled CCD camera (Princeton Instruments) was used for detection. Calibration of the Raman shift for these excitation energies was performed using cyclohexane as a reference. The Raman spectra were invariant with respect to the excitation intensity between 2.5 and 10 $\mathrm{mW}$ (Figure S2).

\section{RESULTS AND DISCUSSION}

Nanostructural and Optical Characterization. Figure 1 shows the TEM micrographs of the uncoated Ag nanocubes (i) and the $\mathrm{Ag} @ \mathrm{CeO}_{2}$ core@shell nanocubes with different ceria shells (ii-iv), for cubes with an edge length of $47 \pm 7 \mathrm{~nm}$ (A) and $71 \pm 9 \mathrm{~nm}(\mathrm{~B})$. For amounts of ceria insufficient to coat the $\mathrm{Ag}$ nanocubes fully, we observe an incomplete coverage with small $\mathrm{CeO}_{2}$ crystallites (ii); otherwise, conformal $\mathrm{CeO}_{2}$ shells with tunable thickness are formed (iii and iv). See Table S1 for specific values for each sample and Figure $S 3$ for additional TEM and SEM micrographs showing no larger structures. There is no significant difference in the size of the isolated ceria crystallites between the 47 and $71 \mathrm{~nm} \mathrm{Ag} \mathrm{nanocubes} \mathrm{coated} \mathrm{with} \mathrm{ceria,} \mathrm{with}$ $2.6 \pm 0.3$ and $2.3 \pm 0.2 \mathrm{~nm}$ sized crystallites, respectively. Aggregated crystallites form the thicker ceria shells, resulting in a relatively conformal coating of the Ag nanocubes. The TEM micrographs show that the ceria shells are porous, that is, have voids. This is most likely due to the absence of a chelating agent which would slow down the deposition of ceria. ${ }^{38}$ XPS reveals a $\mathrm{Ce}^{3+} / \mathrm{Ce}^{4+}$ ratio of 0.09 (see Figure S4 and Table S2). The coverage has been determined from the simulated and experimental extinction properties in different solvents (see below). A summary of shell thicknesses and coverages is provided in Table S1.

Figure 2 presents the UV-vis spectra of the uncoated and coated $47 \mathrm{~nm}$ (A) and $71 \mathrm{~nm}$ (B) Ag nanocubes. The silver interband transition around $300 \mathrm{~nm}$ red-shifts with $\mathrm{CeO}_{2}$ shell thickness; this is likely due to interband scattering, ${ }^{48}$ and, in particular, for the thickest shells, possibly from the $\mathrm{CeO}_{2}$ bandgap $(3.6 \mathrm{eV}, 344 \mathrm{~nm}) .{ }^{49,50}$ The red-shift of all the spectral features with increased $\mathrm{CeO}_{2}$ shell thickness is due to an increase in local index of refraction, as the plasmonic modes are highly sensitive to the local environment. ${ }^{15} \mathrm{CeO}_{2}$ has a refractive index between 2.2 and 2.8 in the visible regime, ${ }^{49,50}$ significantly higher than the refractive index of water. The mode centered at $348 \mathrm{~nm}$ observed for uncoated 47 and $71 \mathrm{~nm} \mathrm{Ag}$ cubes is localized on the face of the cube ${ }^{17}$ and is highly sensitive to the sharpness of the corners. ${ }^{51}$ The retention of this mode during coating suggests that no significant etching of the corners occurs. The mode at $390 \mathrm{~nm}$ ( $47 \mathrm{~nm}$ cubes) and $405 \mathrm{~nm}$ ( $71 \mathrm{~nm}$ cubes) is localized on the edges, while the intense, lowest energy mode at $460 \mathrm{~nm}$ ( $47 \mathrm{~nm}$ cubes) and $485 \mathrm{~nm}$ ( $71 \mathrm{~nm}$ cubes) is localized at the corners of the cube. ${ }^{17}$ The latter mode is strongly dependent on the cube edge length and is the most sensitive to a change in local index of refraction. ${ }^{16,17}$ The broad band around $700 \mathrm{~nm}$ for the $47 \mathrm{~nm}$ silver nanocubes with a $2.6 \mathrm{~nm}$ shell, and to a low extent those around $750 \mathrm{~nm}$ for the $71 \mathrm{~nm}$ silver nanocubes with a $2.3 \mathrm{~nm}$ shell, is most likely due to mild aggregation. It is worth noting that no new electronic transitions indicative of direct $\mathrm{CT}$ are observed.

The ceria shell coverage, while accounting for the voids in the shell, has been determined by correlating experimental and simulated changes in the UV-vis spectrum induced by a change in the local refractive index by using (i) different solvents (Figure S5) and (ii) changing the ceria shell thickness. The volume fraction of the shell $\left(\varphi_{i}\right)$ depends on the shell coverage $(\eta)$ and the distance-dependent refractive index sensitivity (RIS $(t)$, with $t$ the distance) according to: 
A

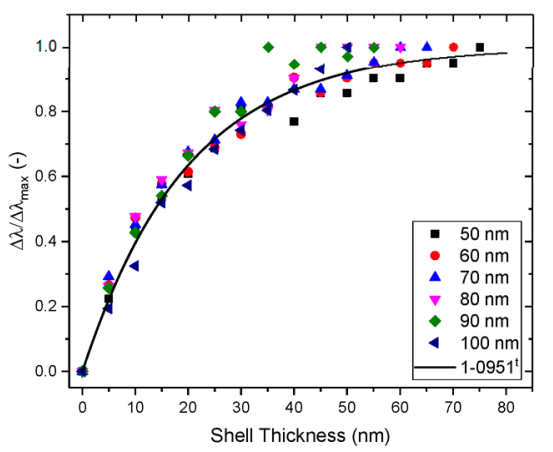

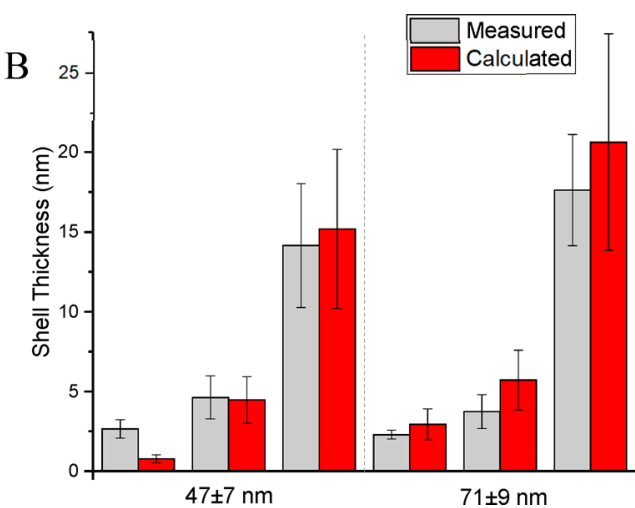

Figure 3. (A) Simulated peak shift of the corner mode with respect to $n$ for cubes of 50-100 nm, normalized from a pure solvent to a pure shell system, including a fit to RIS $(t)=(0.951 \pm 0.009)^{t}$, with $t$ the shell thickness. (B) Comparison of the shell thickness determined by TEM and UV-vis using the results from FDTD and the average coverage, giving statistically similar results.
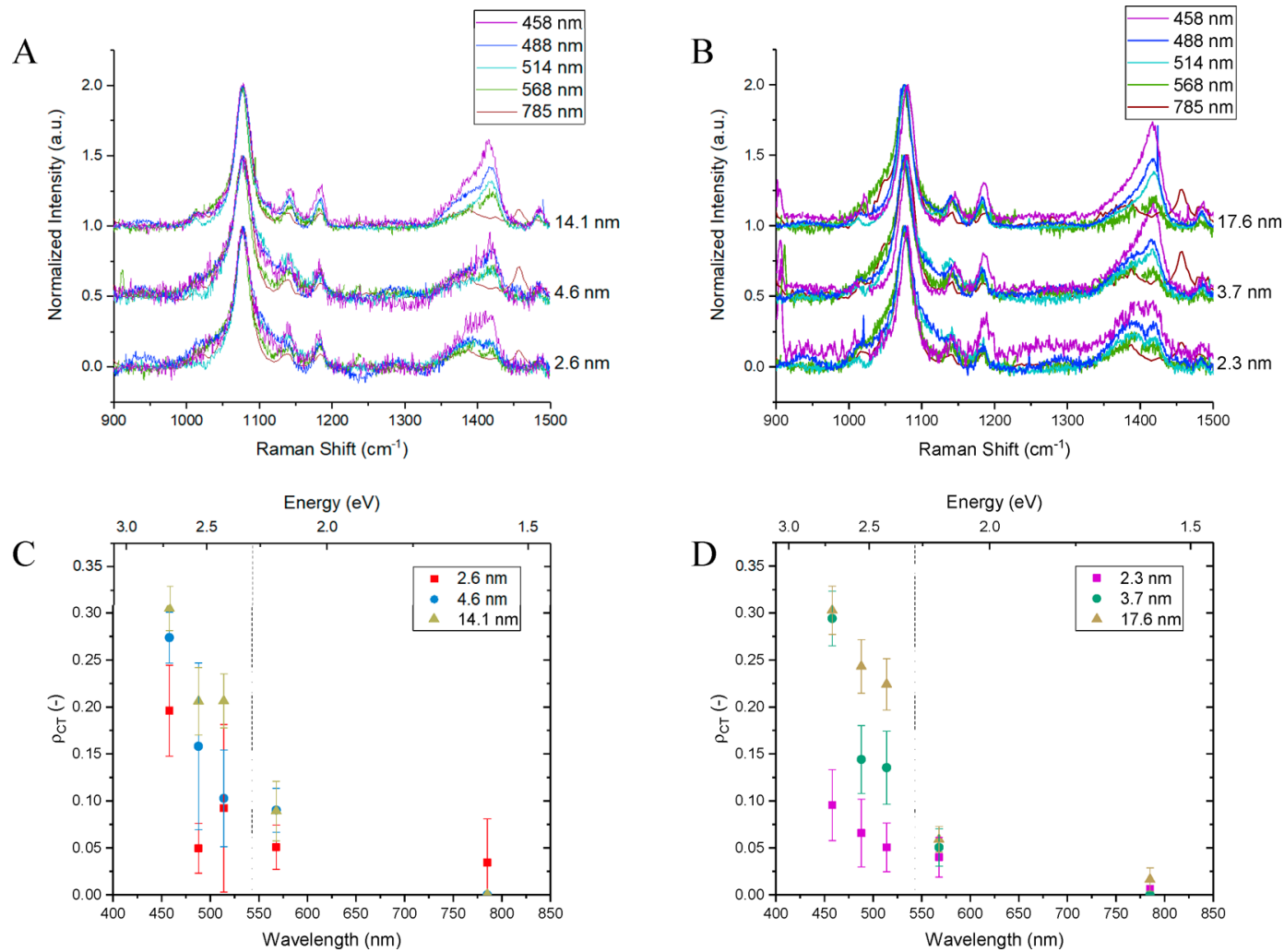

Figure 4. Raman spectra of 4-MBA between silver and ceria for (A) $47 \mathrm{~nm}$ and (B) $71 \mathrm{~nm}$ silver nanocubes, normalized to the intensity of the 1070

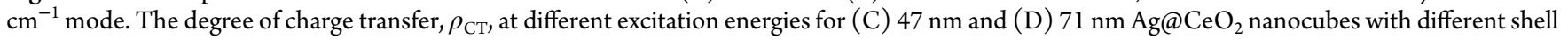
thicknesses; the dashed vertical line is at $543 \mathrm{~nm}$, the wavelength of light needed to excite an electron from the silver Fermi level to the 4-MBA LUMO. The $\rho_{\mathrm{CT}}$ was calculated by using the variance of the $1415 \mathrm{~cm}^{-1}$ mode as compared to the $1070 \mathrm{~cm}^{-1}$ mode.

$$
\varphi_{i}=\frac{\int_{0}^{t} \eta R I S(t) \mathrm{d} t}{\int_{0}^{\infty} R I S(t) \mathrm{d} t}=\eta(1-\operatorname{RIS}(t))
$$

due to the $|E|^{2}$ profile around the plasmonic nanostructure. ${ }^{15}$ The value of $\varphi_{i}$ has been determined by using the LorentzLorenz equation: ${ }^{52}$

$$
\frac{n_{\mathrm{eff}}^{2}-1}{n_{\mathrm{eff}}^{2}+2}=\sum \varphi_{i} \frac{n_{i}^{2}-1}{n_{i}^{2}+2}
$$

where $n_{\text {eff }}$ is the effective refractive index, a function of the $i$ th component's refractive index $n_{i}$ and $\varphi_{i}$ the volume fraction. There are only two components considered for this system: the shell and solvent as the PVP/4-MBA capping agent is a constant and needed to prevent aggregation. The effective refractive index of the Ag cubes has been determined from RIS $(t)$ by dispersing uncoated $\mathrm{Ag}$ cubes in solvents of different refractive indexes and determining the peak shift of the corner mode relative to $n$ (see Figure S5).

Figure 3A shows the effect of ceria shell thickness on the peak shift of the corner mode, simulated by FDTD for cubes with an edge length ranging from 50 to $100 \mathrm{~nm}$ : for an edge length $\leq 90$ $\mathrm{nm}, \mathrm{RIS}(t)=(0.951 \pm 0.009)^{t}$ (see fit included in Figure $3 \mathrm{~A}$ and Figure S6). This model of RIS $(t)$ has been validated by comparing all shell thicknesses as determined by TEM with simulated values (Figure 3B). Applying eq 1 yields an average 
shell coverage $\eta=0.67 \pm 0.25$ (specific values for all samples are shown in Table S1), allowing quantification of the shell thickness through UV-vis spectra alone in future synthesis of $\mathrm{Ag} @ \mathrm{CeO}_{2}$ nanocubes.

Photoinduced Charge Transfer. The 4-MBA linker has been used as a SERS probe for photoinduced CT and facilitates examination of the role of photoexcitation wavelength and core@shell dimensions. The degree of CT $\left(\rho_{\mathrm{CT}}\right)$ has been determined using the theory developed by Lombardi and Birke. Equation 3 compares the selective enhancement of the nontotally symmetric vibrational modes, which are enhanced by CT, relative to totally symmetric modes which are only enhanced by the LSPR. A value of 0.5 implies equal Raman enhancement from LSPR and CT: ${ }^{41}$

$$
\rho_{\mathrm{CT}}(k)=\frac{I^{k}(\mathrm{CT})-I^{k}(\mathrm{LSPR})}{I^{k}(\mathrm{CT})+I^{0}(\mathrm{LSPR})}
$$

The parameter $k$ is an index identifying individual molecular bands in the Raman spectrum. $I^{k}$ (LSPR) is the intensity of line (k) where only the LSPR contributes to the SERS enhancement, while $I^{0}$ (LSPR) denotes the intensity of a chosen totally symmetric line, also with only LSPR contribution. $I^{k}(\mathrm{CT})$ is the intensity of a non-totally symmetric mode where both LSPR and CT contribute. For a non-totally symmetric line $I^{k}$ (LSPR) will normally be small or zero, reducing eq 3 into

$$
\rho_{\mathrm{CT}}(k)=\frac{\frac{I^{k}(\mathrm{CT})}{I^{0}(\mathrm{LSPR})}}{1+\frac{I^{k}(\mathrm{CT})}{I^{0}(\mathrm{LSPR})}}
$$

This method is only capable of observing direct CT that involves the 4-MBA linker. It is not possible to observe any CT directly from silver to ceria bypassing the 4-MBA. Additionally, there is no information regarding the directionality of the process. However, given the lack of light absorption by the ceria or 4MBA and the strong absorption by the silver, it can be concluded that CT originating from light absorbed by the silver is the most likely mechanism.

Figure 4 shows the Raman spectra at various excitation wavelengths for the $47 \mathrm{~nm}$ (A) and $71 \mathrm{~nm}$ (B) Ag nanocubes with 4-MBA linker and different ceria shell thicknesses. The Raman spectra are invariant to the laser intensity between 2.5 and $10 \mathrm{~mW}$ (see Figure S2). The mode at $1070 \mathrm{~cm}^{-1}$ used for normalization of the Raman spectra is the $\mathrm{C}-\mathrm{S}$ stretch and a symmetric ring breathing mode $(\nu \mathrm{CS} \text { and } 1)^{53-55}$ and has $\mathrm{A}_{1}$ character. The assignment of the 1140 and $1180 \mathrm{~cm}^{-1}$ modes is in debate-however, not the modes at 1370 and $1415 \mathrm{~cm}^{-1}$ which are of $B_{2}$ character. ${ }^{54-58}$ Specifically, the $1415 \mathrm{~cm}^{-1}$ mode is the asymmetric ring mode $18 \mathrm{~b}$, and the $1370 \mathrm{~cm}^{-1}$ is assigned to $\beta \mathrm{O}-\mathrm{H}, \nu \mathrm{C}-\mathrm{ph}, 19 \mathrm{a}$, and asymmetric $\nu \mathrm{CO}_{2}$ vibrations. ${ }^{3}-55$ Table 1 summarizes the assignments of the modes. On the basis of these assignments, we have used the $1415 \mathrm{~cm}^{-1}$ mode as $I^{k}(\mathrm{CT})$ and the $1070 \mathrm{~cm}^{-1}$ mode as $I^{0}(\mathrm{LSPR})$ to resolve $\rho_{\mathrm{CT}}$. For quantification of $\rho_{\mathrm{CT}}$, the spectra have been fit with Gaussian functions (see the Supporting Information for details).

An important parameter to address is the 4-MBA layer thickness. In the ideal case a monolayer is present, with the 4MBA molecule likely attached to the Ag core via the thiol group and to the ceria shell via the carboxylic acid group, ${ }^{46}$ implying a subnanometer 4-MBA layer thickness. Multilayer structures may be formed through hydrogen bonding between the thiol and the carboxylic acid groups and by van der Waals interactions.
Table 1. Raman Mode and Symmetry Assignments from the Literature for 4-MBA

\begin{tabular}{clcl}
$\begin{array}{c}\text { wavenumber } \\
\left(\mathrm{cm}^{-1}\right)\end{array}$ & \multicolumn{1}{c}{ band assignment ${ }^{a}$} & ref \\
1070 & $\nu \mathrm{CS}$ and 1 & $\mathrm{~A}_{1}$ & $55,57,65$ \\
1140 & $9 \mathrm{a}+\gamma \mathrm{OH}$ & $\mathrm{A}_{1}$ & 55 \\
& $\delta \mathrm{CH}+\nu 15$ & $\mathrm{~B}_{2}$ & 57,65 \\
1180 & $\beta \mathrm{OH}+9 \mathrm{a}$ & $\mathrm{B}_{2}$ & 55 \\
& $\delta \mathrm{CH}+9 \mathrm{a}$ & $\mathrm{A}_{1}$ & 57 \\
& $\nu \mathrm{CH}$ & $\mathrm{A}_{1}$ & 57,59 \\
1370 & $\beta \mathrm{OH}+\nu \mathrm{C}-\mathrm{ph}+19 \mathrm{a}$ and & $\mathrm{B}_{2}$ & 55 \\
\multirow{2}{*}{1415} & $\nu_{\mathrm{as}} \mathrm{CO}_{2}$ & $\mathrm{~B}_{2}$ & 55,59 \\
& $18 \mathrm{~b}$ & $\mathrm{~B}_{2}$ & 57 \\
& $\beta \mathrm{OH}+\nu \mathrm{C}-\mathrm{ph}+19 \mathrm{a}$ and & & 59,63 \\
& $\nu_{\mathrm{as}} \mathrm{CO}_{2}$ & & 55 \\
1600 & $\nu \mathrm{COO}$ & $\mathrm{A}_{1}$ & 59,63
\end{tabular}

${ }^{a} \nu$ : symmetric stretching; $\nu_{\text {as }}$ : asymmetric stretching; $\beta$ : bending; $\delta$ : deformation.

However, such a multilayer structure would show a $\beta \mathrm{OH}$ Raman mode which would vary between an incomplete shell to the complete shell; that is, variation of the $1180 \mathrm{~cm}^{-1}$ mode and the $1370 \mathrm{~cm}^{-1}$ mode. ${ }^{46}$ As such effect is absent in the Raman spectra (Figure 4A,B), it can be concluded that a multilayer of 4-MBA is unlikely, which is further supported by the TEM experiments (Figure 1) unable to resolve the 4-MBA linker. Incomplete coverage of the Ag surface can however not be excluded, although no PVP Raman signals have been observed.

Figure $4 \mathrm{C}$ shows $\rho_{\mathrm{CT}}$ as a function of excitation wavelength for the $47 \mathrm{~nm} \mathrm{Ag}$ nanocubes with three different ceria shell thicknesses. The measurements at $785 \mathrm{~nm}$ excitation have $1 \%$ ethanol, which gives rise to the extraneous peak at $1460 \mathrm{~cm}^{-1}$. For both nanocube edge lengths, the most pronounced effect on the intensities of the 1370 and $1415 \mathrm{~cm}^{-1}$ modes is observed at 488 and $458 \mathrm{~nm}$ excitation. At $785 \mathrm{~nm}$ excitation $\rho_{\mathrm{CT}}$ is close to zero for all three ceria shell thicknesses, as can be expected for photon energies significantly lower than both the LSPR and a potential Ag to 4-MBA electronic transition. The $\rho_{\mathrm{CT}}$ value significantly increases on decreasing the excitation wavelength, with a notable increase at $514 \mathrm{~nm}$ and shorter wavelengths. In addition, $\rho_{\mathrm{CT}}$ is higher for thicker ceria shells. Figure $4 \mathrm{D}$, presenting $\rho_{\mathrm{CT}}$ values for the $71 \mathrm{~nm} \mathrm{Ag}$ nanocubes coated with different ceria shell thicknesses, shows similar trends. The absence of a band at $1415 \mathrm{~cm}^{-1}$ for Ag cubes coated with 4-MBA only (Figure S7) demonstrates that the ceria shell is crucial for the CT process.

Figure 5 shows an energy level diagram ${ }^{24,59}$ and the proposed CT mechanism. If the CT were to occur through PICTT, CID, or indirect $\mathrm{CT}$, then it would be expected that $\rho_{\mathrm{CT}}$ would follow the LSPR absorption of the silver core. ${ }^{60}$ Given that no LSPR dependency is observed here, $\mathrm{CT}$ is most likely dominated by the DICTT mechanism. The increase in $\rho_{\mathrm{CT}}$ at $514 \mathrm{~nm}$ and shorter wavelengths can be assigned to $\mathrm{CT}$ of an electron from the Ag core to the LUMO of the 4-MBA, which is allowed for photon energies $>2.28 \mathrm{eV}$ (i.e., $<543 \mathrm{~nm}$ ) through the DICTT mechanism. The increase with photon energy likely originates from the increasing probability for CT from an electron at a greater depth in the Fermi sea; on average, half the photon energy is left in the hole formed on photoexcitation. ${ }^{6}$ Indeed, at $785 \mathrm{~nm}$ excitation CT is insignificant for all core@shell geometries. However, all $\rho_{\mathrm{CT}}$ values at $568 \mathrm{~nm}$ excitation exceed 


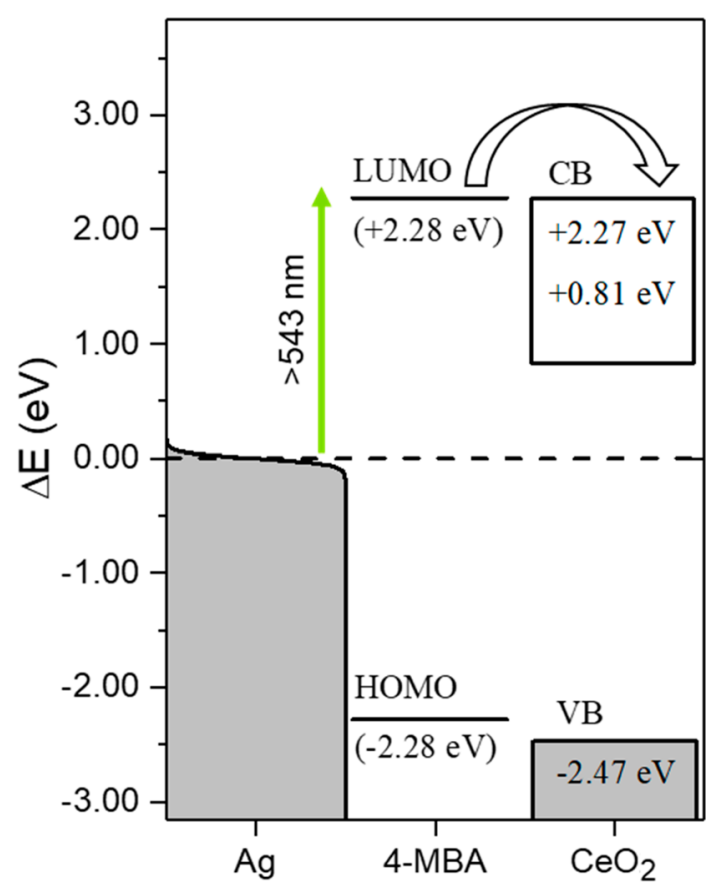

Figure 5. Energy diagram and proposed mechanisms of light-induced $\mathrm{CT}$, the black dashed line is the Fermi energy of silver used as a reference. ${ }^{59,66}$ Photoexcitation could promote an electron from the $\mathrm{Ag}$ core to the 4-MBA linker, followed by electron transfer from the 4-MBA to the $\mathrm{CeO}_{2}$. The latter process may result in the formation of an oxygen vacancy $\left(\mathrm{O}_{\mathrm{vac}}\right)$.

those at $785 \mathrm{~nm}$, indicating some CT, although inefficient. The photon energy at $568 \mathrm{~nm}$ light $(2.18 \mathrm{eV})$ is $0.1 \mathrm{eV}$ below the energy gap between the Ag Fermi level and the LUMO of the 4MBA linker. ${ }^{59}$ Within the thermal distribution of electrons at room temperature, there is a $2 \%$ chance of the state $0.1 \mathrm{eV}$ above the silver Fermi level being populated (Figure S8), which likely accounts for the low $\rho_{\mathrm{CT}}$. Additionally, CT from the 4-MBA HOMO to the silver may occur. While this may have some contribution to $\rho_{\mathrm{CT}}$, it is not expected to demonstrate the trends seen here, as it would lead to relatively constant $\rho_{\mathrm{CT}}$ values for wavelengths shorter than $543 \mathrm{~nm}$, which is not observed.

Aside from this excitation wavelength effect on $\rho_{\mathrm{CT}}$, a second general trend observed is a higher $\rho_{\mathrm{CT}}$ value with increasing ceria layer thickness. This dependency indicates that not only the 4MBA intermediate layer affects the $\mathrm{CT}$ process but also the ceria outer shell. There are statistically significant increases in $\rho_{\mathrm{CT}}$ with a thicker shell through the 4-MBA pathway (Figure 4C,D), which indicate that the electron excited from the $\mathrm{Ag}$ core to the 4-MBA can relax into the $\mathrm{CeO}_{2}$ conduction band (CB). Similar observations were made for a $\mathrm{Au} / 4-\mathrm{MBA} / \mathrm{TiO}_{2}$ system, where the metal to 4-MBA CT occurs, and then the electron is transferred to the semiconductor. ${ }^{60}$ The $\rho_{\mathrm{CT}}$ value for, in particular, the thinnest shells may be limited by charge buildup, which impedes further $\mathrm{CT}^{61}$ This effect likely decreases with increasing shell thickness, explaining the higher $\rho_{\mathrm{CT}}$ observed.

Interestingly, a dependency on the core size is not observed here. In other works, ${ }^{11,60}$ there has been a significant increase in $\rho_{\mathrm{CT}}$ values for excitation wavelengths that were in resonance. Here we observed a $\rho_{\mathrm{CT}}$ dependency purely determined by incident photon energy, apparently independent of the LSPR position, indicating a difference in mechanism (DICTT here, CID in ref 7, and PIRET in ref 8). Additionally, no significant shift of the Raman peaks that could be induced by a strong electric field ${ }^{62,63}$ is observed for the two core sizes studied here. Only a slight shift is seen in the $1070 \mathrm{~cm}^{-1}$ mode for $\mathrm{Ag}$ cubes without a ceria shell (Figure S7), indicative of a difference in hydrogen bonding. ${ }^{64}$ This $\mathrm{Ag} @ \mathrm{CeO}_{2}$ system merits further studies through photocatalytic activity testing and ultrafast spectroscopy to investigate photoinduced formation of oxygen vacancies.

\section{CONCLUSIONS}

This study focuses on the relation between the dimensions of $\mathrm{Ag} @ \mathrm{CeO}_{2}$ nanocubes and the efficiency and mechanism of light-induced CT. A 4-MBA linker between core and shell has been used as a probe for light-induced direct CT by Raman spectroscopy. The ceria shell coverage has been determined with FDTD simulations, which describe the distance-dependent refractive index sensitivity, and UV-vis spectroscopy.

The degree of photoinduced CT has been determined by comparing the intensities of totally symmetric and non-totally symmetric modes. For all $\mathrm{Ag} @ \mathrm{CeO}_{2}$ nanocubes investigated, the degree of CT increases with decreasing excitation wavelength, with a pronounced increase around $514 \mathrm{~nm}$. This trend is fully explicable through a direct CT mechanism from the silver Fermi level to the 4-MBA LUMO. Interestingly, there was no trend seen relating to the core size or to the extinction of the silver nanocube core.

A second general trend observed is a higher CT yield with increasing ceria shell thickness, indicating further electron transfer from the 4-MBA to the ceria shell. It may be that electron transfer from 4-MBA to $\mathrm{CeO}_{2}$ becomes more likely with increasing shell thickness. As this process could lead to formation of oxygen vacancies, this shows promise for photocatalytic application. These results provide deeper understanding of light-induced charge transfer in plasmonic@ semiconductor nanocubes.

\section{ASSOCIATED CONTENT}

\section{Supporting Information}

The Supporting Information is available free of charge at https://pubs.acs.org/doi/10.1021/acsami.9b18393.

X-ray diffraction patterns of the ceria-coated Ag nanocubes; additional Raman, TEM, and SEM data; a representative FDTD simulation; XPS analysis; supportive data on wavelength-dependent excitation; and discussion of the Raman spectral analysis (PDF)

The MATLAB script used to analyze Raman spectra (TXT)

\section{AUTHOR INFORMATION}

\section{Corresponding Author}

*E-mail: j.m.huijser@utwente.nl. Phone: +31 534894449.

ORCID

Devin B. O’Neill: 0000-0001-8833-7772

Anatoli Ianoul: 0000-0002-4933-9836

Guido Mul: 0000-0001-5898-6384

Annemarie Huijser: 0000-0003-0381-6155

Notes

The authors declare no competing financial interest.

\section{ACKNOWLEDGMENTS}

This research is financed by The Netherlands Organisation for Scientific Research (NWO, project number 731.015.603) and 
supported by BASF, SABIC, and SASOL. Robert Meijer is acknowledged for technical support and Piotr Krzywda for help with the SEM analysis.

\section{ABBREVIATIONS}

$\mathrm{CB}$, conduction band; CID, chemical interface dampening; CT, charge transfer; DICTT, direct interface charge transfer transition; FDTD, finite-difference time domain; HOMO, highest occupied molecular orbital; LSPR, localized surface plasmon resonance; LUMO, lowest unoccupied molecular orbital; 4-MBA, 4-mercaptobenzoic acid; PIRET, plasmoninduced resonant energy transfer; PVP, polyvinylpyrrolidone; SERS, surface-enhanced Raman spectroscopy; $\rho_{\mathrm{CT}}$, degree of charge transfer; VB, valence band.

\section{REFERENCES}

(1) Kubacka, A.; Fernández-García, M.; Colón, G. Advanced Nanoarchitectures for Solar Photocatalytic Applications. Chem. Rev. 2012, 112 (3), 1555-1614.

(2) Gust, D.; Moore, T. A.; Moore, A. L. Solar Fuels via Artificial Photosynthesis. Acc. Chem. Res. 2009, 42 (12), 1890-1898.

(3) Linic, S.; Christopher, P.; Ingram, D. B. Plasmonic-Metal Nanostructures for Efficient Conversion of Solar to Chemical Energy. Nat. Mater. 2011, 10 (12), 911-921.

(4) Cushing, S. K.; Li, J.; Bright, J.; Yost, B. T.; Zheng, P.; Bristow, A. D.; $\mathrm{Wu}, \mathrm{N}$. Controlling Plasmon-Induced Resonance Energy Transfer and Hot Electron Injection Processes in Metal@TiO $\mathrm{TiO}_{2}$ Core-Shell Nanoparticles. J. Phys. Chem. C 2015, 119 (28), 16239-16244.

(5) Li, J.; Cushing, S. K.; Meng, F.; Senty, T. R.; Bristow, A. D.; Wu, N. Plasmon-Induced Resonance Energy Transfer for Solar Energy Conversion. Nat. Photonics 2015, 9 (9), 601-607.

(6) Gieseking, R. L.; Ratner, M. A.; Schatz, G. C. Review of PlasmonInduced Hot-Electron Dynamics and Related SERS Chemical Effects. ACS Symp. Ser. 2016, 1245, 1-22.

(7) Kale, M. J.; Christopher, P. Plasmons at the Interface. Science 2015, 349 (6248), 587-588.

(8) Wu, K.; Chen, J.; McBride, J. R.; Lian, T. Efficient Hot-Electron Transfer by a Plasmon-Induced Interfacial Charge-Transfer Transition. Science 2015, 349 (6248), 632-635.

(9) Boerigter, C.; Aslam, U.; Linic, S. Mechanism of Charge Transfer from Plasmonic Nanostructures to Chemically Attached Materials. ACS Nano 2016, 10 (6), 6108-6115.

(10) Baffou, G.; Quidant, R. Thermo-Plasmonics: Using Metallic Nanostructures as Nano-Sources of Heat. Laser Photon. Rev. 2013, 7 (2), 171-187.

(11) Kale, M. J.; Avanesian, T.; Xin, H.; Yan, J.; Christopher, P. Controlling Catalytic Selectivity on Metal Nanoparticles by Direct Photoexcitation of Adsorbate-Metal Bonds. Nano Lett. 2014, 14 (9), $5405-5412$.

(12) Ahamad, N.; Bottomley, A.; Ianoul, A. Optimizing Refractive Index Sensitivity of Supported Silver Nanocube Monolayers. J. Phys. Chem. C 2012, 116 (1), 185-192.

(13) Wu, X.; Xia, Y.; Huang, Y.; Li, J.; Ruan, H.; Chen, T.; Luo, L.; Shen, Z.; Wu, A. Improved SERS-Active Nanoparticles with Various Shapes for CTC Detection without Enrichment Process with Supersensitivity and High Specificity. ACS Appl. Mater. Interfaces 2016, 8 (31), 19928-19938.

(14) Dutta, S.; Ray, C.; Sarkar, S.; Pradhan, M.; Negishi, Y.; Pal, T. Silver Nanoparticle Decorated Reduced Graphene Oxide (RGO) Nanosheet: A Platform for SERS Based Low-Level Detection of Uranyl Ion. ACS Appl. Mater. Interfaces 2013, 5 (17), 8724-8732.

(15) Willets, K. A.; Van Duyne, R. P. Localized Surface Plasmon Resonance Spectroscopy and Sensing. Annu. Rev. Phys. Chem. 2007, 58 (1), 267-297.

(16) Sherry, L. J.; Chang, S.-H.; Schatz, G. C.; Van Duyne, R. P.; Wiley, B. J.; Xia, Y. Localized Surface Plasmon Resonance Spectroscopy of Single Silver Nanocubes. Nano Lett. 2005, 5 (10), 2034-2038.
(17) Nicoletti, O.; de la Peña, F.; Leary, R. K.; Holland, D. J.; Ducati, C.; Midgley, P. A. Three-Dimensional Imaging of Localized Surface Plasmon Resonances of Metal Nanoparticles. Nature 2013, 502 (7469), $80-84$.

(18) Shelef, M.; McCabe, R. W. Twenty-Five Years after Introduction of Automotive Catalysts: What Next? Catal. Today 2000, 62 (1), 3550.

(19) Montini, T.; Melchionna, M.; Monai, M.; Fornasiero, P. Fundamentals and Catalytic Applications of $\mathrm{CeO}_{2}$-Based Materials. Chem. Rev. 2016, 116 (10), 5987-6041.

(20) Kašpar, J.; Fornasiero, P.; Hickey, N. Automotive Catalytic Converters: Current Status and Some Perspectives. Catal. Today 2003, 77 (4), 419-449.

(21) Liu, Z. E.; Wang, J.; Li, Y.; Hu, X.; Yin, J.; Peng, Y.; Li, Z.; Li, Y.; Li, B.; Yuan, Q. Near-Infrared Light Manipulated Chemoselective Reductions Enabled by an Upconversional Supersandwich Nanostructure. ACS Appl. Mater. Interfaces 2015, 7 (34), 19416-19423.

(22) Jia, H.; Zhu, X. M.; Jiang, R.; Wang, J. Aerosol-Sprayed Gold/ Ceria Photocatalyst with Superior Plasmonic Hot Electron-Enabled Visible-Light Activity. ACS Appl. Mater. Interfaces 2017, 9 (3), 25602571.

(23) Zhang, Y. C.; Li, Z.; Zhang, L.; Pan, L.; Zhang, X.; Wang, L.; Fazal-e-Aleem; Zou, J. J. Role of Oxygen Vacancies in Photocatalytic Water Oxidation on Ceria Oxide: Experiment and DFT Studies. Appl. Catal., B 2018, 224, 101-108.

(24) Ma, R.; Jahurul Islam, M.; Amaranatha Reddy, D.; Kim, T. K. Transformation of $\mathrm{CeO}_{2}$ into a Mixed Phase $\mathrm{CeO}_{2} / \mathrm{Ce}_{2} \mathrm{O}_{3}$ Nanohybrid by Liquid Phase Pulsed Laser Ablation for Enhanced Photocatalytic Activity through Z-Scheme Pattern. Ceram. Int. 2016, 42 (16), 1849518502.

(25) Goguet, A.; Meunier, F. C.; Tibiletti, D.; Breen, J. P.; Burch, R. Spectrokinetic Investigation of Reverse Water-Gas-Shift Reaction Intermediates over a Pt $/ \mathrm{CeO}_{2}$ catalyst. J. Phys. Chem. B 2004, 108 (52), 20240-20246.

(26) Inerbaev, T. M.; Karakoti, A. S.; Kuchibhatla, S. V. N. T. N. T.; Kumar, A.; Masunov, A. E.; Seal, S. Aqueous Medium Induced Optical Transitions in Cerium Oxide Nanoparticles. Phys. Chem. Chem. Phys. 2015, 17 (9), 6217-6221.

(27) Wang, F.; Shen, Y. R. General Properties of Local Plasmons in Metal Nanostructures. Phys. Rev. Lett. 2006, 97 (20), 1-4.

(28) Singh Sekhon, J.; Verma, S. S. Refractive Index Sensitivity Analysis of Ag, Au, and $\mathrm{Cu}$ Nanoparticles. Plasmonics 2011, 6 (2), 311317.

(29) Cobley, C. M.; Skrabalak, S. E.; Campbell, D. J.; Xia, Y. ShapeControlled Synthesis of Silver Nanoparticles for Plasmonic and Sensing Applications. Plasmonics 2009, 4 (2), 171-179.

(30) Chen, M.; Gu, J.; Sun, C.; Zhao, Y.; Zhang, R.; You, X.; Liu, Q.; Zhang, W.; Su, Y.; Su, H. Light-Driven Overall Water Splitting Enabled by a Photo-Dember Effect Realized on 3D Plasmonic Structures. ACS Nano 2016, 10 (7), 6693-6701.

(31) Yu, L.; Peng, R.; Chen, L.; Fu, M.; Wu, J.; Ye, D. Ag Supported on $\mathrm{CeO}_{2}$ with Different Morphologies for the Catalytic Oxidation of HCHO. Chem. Eng. J. 2018, 334, 2480-2487.

(32) Raudonyte-Svirbutaviciene, E.; Neagu, A.; Vickackaite, V.; Jasulaitiene, V.; Zarkov, A.; Tai, C. W.; Katelnikovas, A. Two-Step Photochemical Inorganic Approach to the Synthesis of $\mathrm{Ag}-\mathrm{CeO}_{2}$ nanoheterostructures and Their Photocatalytic Activity on Tributyltin Degradation. J. Photochem. Photobiol., A 2018, 351, 29-41.

(33) Grabchenko, M. V.; Mikheeva, N. N.; Mamontov, G. V.; Salaev, M. A.; Liotta, L. F.; Vodyankina, O. V. $\mathrm{Ag} / \mathrm{CeO}_{2}$ Composites for Catalytic Abatement of CO, Soot and VOCs. Catalysts 2018, 8 (7), 285.

(34) Chang, S.; Li, M.; Hua, Q.; Zhang, L.; Ma, Y.; Ye, B.; Huang, W. Shape-Dependent Interplay between Oxygen Vacancies and Ag$\mathrm{CeO}_{2}$ interaction in $\mathrm{Ag} / \mathrm{CeO}_{2}$ catalysts and Their Influence on the Catalytic Activity. J. Catal. 2012, 293, 195-204.

(35) Wang, Y.-Y.; Shu, Y.; Xu, J.; Pang, H. Facile One-Step Synthesis of Ag@CeO ${ }_{2}$ Core-Shell Nanospheres with Efficient Catalytic Activity for the Reduction of 4-Nitrophenol. CrystEngComm 2017, 19 (4), 684689. 
(36) Chen, S.-Y.; Tseng, E.; Lai, Y.-T.; Lee, W.; Gloter, A. Interface Interactions and Enhanced Room Temperature Ferromagnetism of Ag@ $\mathrm{CeO}_{2}$ Nanostructures. Nanoscale 2017, 9 (30), 10764-10772.

(37) Wu, L.; Fang, S.; Ge, L.; Han, C.; Qiu, P.; Xin, Y. Facile Synthesis of $\mathrm{Ag} @ \mathrm{CeO}_{2}$ Core-Shell Plasmonic Photocatalysts with Enhanced Visible-Light Photocatalytic Performance. J. Hazard. Mater. 2015, 300 (18), 93-103.

(38) Wang, J. J.; Li, B.; Gu, T.; Ming, T.; Wang, J. J.; Wang, P.; Yu, J. C. (Gold Core) at (Ceria Shell) Nanostructures for Plasmon-Enhanced Catalytic Reactions under Visible Light. ACS Nano 2014, 8 (8), 81528162

(39) Pelli Cresi, J. S.; Spadaro, M. C.; D’Addato, S.; Valeri, S.; Benedetti, S.; di Bona, A.; Catone, D.; Di Mario, L.; O'Keeffe, P.; Paladini, A.; et al. Highly Efficient Plasmon-Mediated Electron Injection into Cerium Oxide from Embedded Silver Nanoparticles. Nanoscale 2019, 11 (21), 10282-10291.

(40) Yang, Y.; Matsubara, S.; Xiong, L.; Hayakawa, T.; Nogami, M. Solvothermal Synthesis of Multiple Shapes of Silver Nanoparticles and Their SERS Properties. J. Phys. Chem. C 2007, 111 (26), 9095-9104.

(41) Lombardi, J. R.; Birke, R. L. A Unified Approach to SurfaceEnhanced Raman Spectroscopy. J. Phys. Chem. C 2008, 112 (14), 5605-5617.

(42) Skrabalak, S. E.; Au, L.; Li, X.; Xia, Y. Facile Synthesis of Ag Nanocubes and Au Nanocages. Nat. Protoc. 2007, 2 (9), 2182-2190.

(43) Bottomley, A.; Prezgot, D.; Ianoul, A. Plasmonic Properties of Silver Nanocube Monolayers on High Refractive Index Substrates. Appl. Phys. A: Mater. Sci. Process. 2012, 109, 869-872.

(44) Garnett, E. C.; Cai, W.; Cha, J. J.; Mahmood, F.; Connor, S. T.; Greyson Christoforo, M.; Cui, Y.; McGehee, M. D.; Brongersma, M. L. Self-Limited Plasmonic Welding of Silver Nanowire Junctions. Nat. Mater. 2012, 11 (3), 241-249.

(45) Tseng, C. H. T.; Paul, B. K.; Chang, C.-H.; Engelhard, M. H. Continuous Precipitation of Ceria Nanoparticles from a Continuous Flow Micromixer. Int. J. Adv. Manuf. Technol. 2013, 64 (1-4), 579586.

(46) Ho, C. H.; Lee, S. SERS and DFT Investigation of the Adsorption Behavior of 4-Mercaptobenzoic Acid on Silver Colloids. Colloids Surf., A 2015, 474, 29-35.

(47) Johnson, P. B.; Christy, R. W. Optical Constants of the Noble Metals. Phys. Rev. B 1972, 6 (12), 4370-4379.

(48) See, K. C.; Spicer, J. B.; Brupbacher, J.; Zhang, D.; Vargo, T. G. Modeling Interband Transitions in Silver Nanoparticle-Fluoropolymer Composites. J. Phys. Chem. B 2005, 109 (7), 2693-2698.

(49) Bruno, G.; Gasparotto, A.; Tondello, E.; Barreca, D.; Losurdo, M. Nanostructure and Optical Properties of $\mathrm{CeO}_{2}$ Thin Films Obtained by Plasma-Enhanced Chemical Vapor Deposition. Mater. Sci. Eng., C 2003, 23 (6-8), 1013-1016.

(50) Oh, T. S.; Tokpanov, Y. S.; Hao, Y.; Jung, W.; Haile, S. M. Determination of Optical and Microstructural Parameters of Ceria Films. J. Appl. Phys. 2012, 112 (10), 103535.

(51) Grillet, N.; Manchon, D.; Bertorelle, F.; Bonnet, C.; Broyer, M.; Cottancin, E.; Lermé, J.; Hillenkamp, M.; Pellarin, M. Plasmon Coupling in Silver Nanocube Dimers: Resonance Splitting Induced by Edge Rounding. ACS Nano 2011, 5 (12), 9450-9462.

(52) Aspnes, D. E. Local field Effects and Effective medium Theory: A Microscopic Perspective. Am. J. Phys. 1982, 50 (8), 704-709.

(53) Preuss, M.; Bechstedt, F. Vibrational Spectra of Ammonia, Benzene, and Benzene Adsorbed on Si (001) by First Principles Calculations with Periodic Boundary Conditions. Phys. Rev. B: Condens. Matter Mater. Phys. 2006, 73 (15), 155413.

(54) Andrejeva, A.; Gardner, A. M.; Tuttle, W. D.; Wright, T. G. Consistent Assignment of the Vibrations of Symmetric and Asymmetric Para -Disubstituted Benzene Molecules. J. Mol. Spectrosc. 2016, 321 (4), 28-49.

(55) Li, R.; Lv, H.; Zhang, X.; Liu, P.; Chen, L.; Cheng, J.; Zhao, B. Vibrational Spectroscopy and Density Functional Theory Study of 4Mercaptobenzoic Acid. Spectrochim. Acta, Part A 2015, 148, 369-374.

(56) Xue, X.; Ji, W.; Mao, Z.; Mao, H.; Wang, Y.; Wang, X.; Ruan, W.; Zhao, B.; Lombardi, J. R. Raman Investigation of Nanosized $\mathrm{TiO}_{2}$ :
Effect of Crystallite Size and Quantum Confinement. J. Phys. Chem. C 2012, 116 (15), 8792-8797.

(57) Guo, L.; Zhang, X.; Li, P.; Han, R.; Liu, Y.; Han, X.; Zhao, B. Surface-Enhanced Raman Scattering (SERS) as a Probe for Detection of Charge-Transfer between $\mathrm{TiO}_{2}$ and CdS Nanoparticles. New J. Chem. 2019, 43 (1), 230-237.

(58) Chen, L.; Sun, H.; Zhao, Y.; Zhang, Y.; Wang, Y.; Liu, Y.; Zhang, X.; Jiang, Y.; Hua, Z.; Yang, J. Plasmonic-Induced SERS Enhancement of Shell-Dependent Ag@ $@ \mathrm{Cu}_{2} \mathrm{O}$ Core-Shell Nanoparticles. RSC Adv. 2017, 7 (27), 16553-16560.

(59) Zhang, X.; Yu, Z.; Ji, W.; Sui, H.; Cong, Q.; Wang, X.; Zhao, B. Charge-Transfer Effect on Surface-Enhanced Raman Scattering (SERS) in an Ordered Ag NPs/4-Mercaptobenzoic Acid/TiO 2 System. J. Phys. Chem. C 2015, 119 (39), 22439-22444.

(60) Han, R.; Song, W.; Wang, X.; Mao, Z.; Han, X. X.; Zhao, B. Investigation of Charge Transfer at the $\mathrm{TiO}_{2}-\mathrm{MBA}$-Au Interface Based on Surface-Enhanced Raman Scattering: SPR Contribution. Phys. Chem. Chem. Phys. 2018, 20 (8), 5666-5673.

(61) Sheldon, M.; van de Groep, J.; Brown, A.; Polman, A.; Atwater, H. Plasmoelectric Potentials in Metal. Science 2014, 346 (6211), 828831.

(62) Banik, M.; El-Khoury, P. Z.; Nag, A.; Rodriguez-Perez, A.; Guarrottxena, N.; Bazan, G. C.; Apkarian, V. A. Surface-Enhanced Raman Trajectories on a Nano-Dumbbell: Transition from Field to Charge Transfer Plasmons as the Spheres Fuse. ACS Nano 2012, 6 (11), 10343-10354.

(63) Nie, S.; Emory, S. R. Probing Single Molecules and Single Nanoparticles by Surface-Enhanced Raman Scattering. Science 1997, 275 (5303), 1102-1106.

(64) Wang, Y.; Ji, W.; Sui, H.; Kitahama, Y.; Ruan, W.; Ozaki, Y.; Zhao, B. Exploring the Effect of Intermolecular H-Bonding: A Study on Charge-Transfer Contribution to Surface-Enhanced Raman Scattering of p-Mercaptobenzoic Acid. J. Phys. Chem. C 2014, 118 (19), 1019110197.

(65) Chen, L.; Sun, H.; Zhao, Y.; Zhang, Y.; Wang, Y.; Liu, Y.; Zhang, X.; Jiang, Y.; Hua, Z.; Yang, J. Plasmonic-Induced SERS Enhancement of Shell-Dependent $\mathrm{Ag} @ \mathrm{Cu}_{2} \mathrm{O}$ Core-Shell Nanoparticles. RSC Adv. 2017, 7 (27), 16553-16560.

(66) Esch, F.; Africh, C.; Comelli, G.; Fabris, S.; Fornasiero, P.; Montini, T.; Rosei, R.; Zhou, L. Electron Localization Determines Defect Formation on Ceria Substrates. Science 2005, 309 (5735), 752755 . 